\title{
瞄准乙型肝炎病毒的七寸命门进行精准研究
}

张华堂

重庆市科学技术研究院, 重庆 401123

E-mail: zht@ cast.gov.cn

\section{Aiming at the cccDNA for a cure of $\mathrm{HBV}$ persistence}

\section{Huatang Zhang}

Chongqing Academy of Science and Technology, Chongqing 401123, China

E-mail: zht@cast.gov.cn

doi: 10.1360/TB-2019-0533

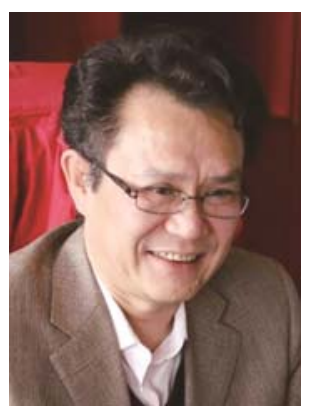

\section{强华堂}

重庆市科学技术研究院生物医 药领域首席科学家、副总工程 师、生物医药与器械研究中心 主任. 牛津大学博士, 2005 年中 国科学院“百人计划”引进国外 杰出人才, 任研究员、博士生导 师、中国科学院知识创新工程 学术带头人、中国实验动物学 会理事、中国微生物学会病毒 学专业委员会委员、兔疫学会 移植免疫学分会委员. 从事肿 瘤、艾滋病与乙型肝炎等重大 疾病的细胞分子生物学基础研 究与医学转化.
迄今, 病毒性肝炎(特别是慢性乙型肝炎)、肝硬化和肝癌仍然是困扰科 学界的世纪性难题。对此，世界卫生组织(WHO)提出了防治病毒性肝炎、“消 除肝炎危害”的“2030 年目标”(https://www.who.int/hepatitis/strategy20162021/ghss-hep/en/). 能否实现这一目标、如何实现这一目标, 特别是从科学 研究的角度, 我们从事乙肝病毒研究的学者, 怎样才能为促成这一目标的实 现做出可能的贡献? 目前病毒性肝炎研究的现状到底如何、关键的瓶颈问题 究竟在哪里、寻求突破的靶点靶标可能在哪里, 以及如何实现期待已久的重 大突破, 是启动本专辑的原始动因之一.

当然, 从人口健康的角度谈清除肝炎的危害和从学术研究的角度谈根 除病毒本身, 在概念和判定指标上是有明确区别的. 简单地说, 能否从根本 上清除乙型肝炎病毒( HBV), 在于我们能否清除其急性感染之后长期、稳定、 持续地滞留或残留在细胞核内的共价闭环DNA(cccDNA). cccDNA 的形成是 $\mathrm{HBV}$ 生命周期中最为关键的环节。不管是临床报告、还是实验室研究，也无 论是抗原、还是核酸水平的检测, 目前的病毒学指标, 包括 $\mathrm{HBs} A \mathrm{Ag} 、 \mathrm{HB} \mathrm{Ag}$ 、 $\mathrm{HBc}$ 相关蛋白、病毒载量/拷贝数、RNA 等, 最终都归于 $\operatorname{cccDNA}$ 的残留、 修饰、变异、转录和复制的多少、快慢的循环以及周而复始的再循环 ${ }^{[1]}$. cccDNA 存在与复制是 HBV 携带状态与慢性乙肝的本质和固有标志, 是乙 肝防控的根本, 是 $\mathrm{HBV}$ 的七寸和命门.

本专辑的基本定位不在于彻底、全面深入地回看乙肝医学史的惨痛与惨 烈或乙型肝炎已给人类带来的灾害 ${ }^{[2]}$, 也不在于系统荣耀地展示乙肝病毒研 究的艰辛、艰难 ${ }^{[3]}$, 以及相关科学研究与医学转化已经对人类健康与疾病控 制所做出的突出贡献和辉煌成就 ${ }^{[4]}$. 本专辑的初衷在于, 在我们的读者群中, 进一步“投石问路”式地明确和强调 HBV cccDNA 的极端重要性, 以便从研 究现状、可能的方向、非凡的难度和突而未破的症结等几个方面进行思考和 探索.

本专辑借中国微生物学会病毒学专业委员会青年委员会核心成员组织 学术活动的契机, 邀请奋战在 HBV 研究最前线的部分“较为年轻”的骨千分 
子和中坚力量分享他们相关的研究经历以及他们的观点和看法. 本专辑的 9 篇进展与述评 ${ }^{[5 \sim 13]}$, 按照从模型、 到机制 ${ }^{[8 \sim 11]}$ 、再到药物治疗和基因编辑 ${ }^{[12,13]}$ 的逻辑和主线, 拟从不同的角度、深度和高度阐释 HBV cccDNA 的形成、模拟、调控, 以及进行治疗和根除的可能性及其研究方法和技术手段.

首先, 3 篇进展分别从 HBV cccDNA 体外细胞实验模型及体内代谢特征 ${ }^{[5]}$ 、人源化小鼠的应 用 $^{[6]}$ 和 $\mathrm{HBeAg}$ 阴性的 HBV cccDNA 小鼠模型的建立与应用前景 ${ }^{[7]}$ 等三个重要方面总结评价了体内外模型研究的现状和前景, 涵盖了产生与制备 HBV cccDNA 的技术手段、细胞与小鼠品系的选择及免疫状态的差别等多方面的思路、经 验和比较, 同时也展示了 HBV 体内外动物模型研究的复杂性、高难度以及对模型研究的高要求和高期待. \# 庸讳言, 简单、实用的体内外模型的遗之一直是严重制约乙肝药物与疫苗研究的瓶颈问题之一, 但同时也提 示, 建立体内外模型各环节的每一点技术进步, 都可能对推展全面研究乙肝的技术体系有重要的价值. 比如, 我们克隆制备了 HBeAg 阴性的 cccDNA, 并在免疫健全的常规小鼠体内建立了可与通常的 HBeAg 阳性病毒 匹配的长期携带模型, 时长超过半年(26 周) 以上 ${ }^{[7]}$, 对研究慢性乙肝的自然史和 $\mathrm{HBeAg}$ 阴性的慢性乙肝可能 具有特别的重要意义. 接下来的机理和新药研究以及基因编辑等进展和述评 ${ }^{[8 \sim 13]}$ 无须赘述, 和前面的模型研 究一起, 请专家学者们汶览、品读和批评. 最后, 本专辑仍有进一步扩充的较大空间, 特别是有关在定性和定 量两个方面简易快捷、特异精准地直接测检 cccDNA 的可靠方法和实验体系, 敬请参见 Zhong 等人 ${ }^{[14]}$, Li 等 人 $^{[15]}$ 和 $\mathrm{GaO}$ 等人 ${ }^{[16]}$ 的相关工作.

希望本专辑能够吸引更多的专家学者同我们一道, 瞄准 HBV 的本源性的固有标志和七寸命门 cccDNA, 开展更新、更高的精准研究, 实现以清除病毒为目标的精准打击.

\section{参考文献}

1 Guo J T, Guo H. Metabolism and function of hepatitis B virus cccDNA: Implications for the development of cccDNA-targeting antiviral therapeutics. Antiviral Res, 2015, 122: 91-100

2 Gerlich W H. Medical virology of hepatitis B: how it began and where we are now. Virol J, 2013, 10: 239

3 Block T M, Alter H J, London W T, et al. A historical perspective on the discovery and elucidation of the hepatitis B virus. Antiviral Res, 2016, 131: 109-123

4 Gish R G, Given B D, Lai C L. Chronic hepatitis B: Virology, natural history, current management and a glimpse at future opportunities. Antiviral Res, 2015, 121: 47-58

5 Zhu Y F, Su Y, Deng Q. In vitro experimental models for the study of hepatitis B virus cccDNA (in Chinese). Chin Sci Bull, 2019, 64: 3061-3069 [朱园飞, 苏瑜, 邓强. 乙型肝炎病毒 cccDNA 体外实验模型研究进展. 科学通报, 2019, 64: 3061-3069]

6 Ren X N, Zhou X H. Humanized mouse models for human viral hepatitis and related liver diseases (in Chinese). Chin Sci Bull, 2019, 64: 3070-3076 [任晓楠, 周晓辉. 人源化小鼠模型在病毒性肝炎等肝脏疾病研究中的应用. 科学通报, 2019, 64: 3070-3076]

7 Liu Y, Zhang H T, Lai G Q. The present situation and prospect of research on HBeAg-negative mouse model (in Chinese). Chin Sci Bull, 2019, 64: 3077-3083 [刘阳, 张华堂, 赖国旗. HBeAg 阴性小鼠模型的研究现状与展望. 科学通报, 2019, 64: 3077-3083]

8 Yuan Y F, Chen X W, Wu C C. Epigenetic modification and regulation of HBV cccDNA (in Chinese). Chin Sci Bull, 2019, 64: 3084-3090 [袁一菲, 陈新文, 吴春晨. HBV cccDNA 的表观遗传学修饰及调控. 科学通报, 2019, 64: 3084-3090]

9 He R, Liu S. Host factors involved in HBV cccDNA formation and transcription (in Chinese). Chin Sci Bull, 2019, 64: 3091-3100 [贺锐, 刘实. 宿主因子参与 HBV cccDNA 形成和转录. 科学通报, 2019, 64: 3091-3100]

10 Guo Y D, Wang J W, Yuan S, et al. The regulation of HBx in hepatitis B virus replication (in Chinese). Chin Sci Bull, 2019, 64: 3101-3108 [郭艳丹, 汪静雯, 袁森, 等. HBx 对 HBV 复制的调控. 科学通报, 2019, 64: 3101-3108]

11 Wang J W, Sun G H. The function of autophagy in diseases caused by hepatitis B virus (in Chinese). Chin Sci Bull, 2019, 64: 3109-3122 [汪静雯, 孙桂鸿. 自噬在乙肝病毒引起的疾病中的功能. 科学通报, 2019, 64: 3109-3122]

12 Xu Z C, Zhao K T, Jiang Y A, et al. Development of antiviral drugs against hepatitis B virus (in Chinese). Chin Sci Bull, 2019, 64: 3123-3141 [徐在超, 赵凯涛, 江应安, 等. 乙型肝炎抗病毒药物研究进展. 科学通报, 2019, 64: 3123-3141]

13 Yan K, Feng J P, Xiong Y, et al. Application of CRISPR-Cas9 gene editing to treat HBV (in Chinese). Chin Sci Bull, 2019, 64: 3142-3150 [严鲲, 冯姜澎, 熊勇, 等. CRISPR-Cas9 基因编辑技术在乙型肝炎病毒治疗中的应用. 科学通报, 2019, 64: 3142-3150]

14 Zhong Y, Han J, Zou Z, et al. Quantitation of HBV covalently closed circular DNA in micro formalin fixed paraffin-embedded liver tissue using rolling circle amplification in combination with real-time PCR. Clin Chim Acta, 2011, 412: 1905-1911

15 Li X, Zhao J, Yuan Q, et al. Detection of HBV covalently closed circular DNA. Viruses, 2017, 9: pii: e139

16 Gao Z, Yan L, Li W. A quantitative method for hepatitis B virus covalently closed circular DNA enables distinguishing direct acting antivirals from cytotoxic agents. Antiviral Res, 2019, 168: 197-202 